\title{
Interception of the near space hypersonic weapons based on the partial integrated guidance and control method
}

\author{
Bin $\mathrm{Fu}^{\mathrm{a}}$, Hang $\mathrm{Guo}^{\mathrm{h}}$ and Jie Yan ${ }^{\mathrm{c}}$ \\ College of Astronautics, Northwestern Polytechnical University, Xi'an, China \\ abinfu@mail.nwpu.edu.cn, bjsguoh@mail.nwpu.edu.cn, cjyan@nwpu.edu.cn
}

\begin{abstract}
Keywords: Hypersonic target interception, high order sliding mode control, partial integrated guidance and control, arbitrary order robust exact differentiator.

Abstract. The interception of the hypersonic weapons in near space is the main problem considered in this paper. The designed interceptor is assumed to have much lower speed than its target and very limited maneuverability in the near space atmosphere. To deal with this problem, a two-loop Partial Integrated Guidance and Control (PIGC) scheme is introduced in this design. More specifically, an integrated full state feedback guidance loop and an inner state feedback control loop together provide a quick and stable response to the target maneuver. To realize the PIGC scheme, a Quasi-continuous High Order Sliding Mode controller is employed due to the high order relative degree nature of the PIGC model. Finally, numerical simulation results in three typical engagement scenarios are given to demonstrate the superiority of the proposed method.
\end{abstract}

\section{I.Introduction}

With the rapid development of the hypersonic technology, hypersonic weapons will surly have an important role in the future tactic and strategic strike missions. On the other side, defense technology should also take further steps to tackle those future threats. Hypersonic weapons, differ from the traditional ballistic missile, can fly at a near space altitude with a hypersonic speed up to 10-20Ma, and a maneuverability up to $9 g[1]$. At the near space altitude, interceptor with aerodynamic rudders will not be able to perform a 40-50g maneuver as ordinary air-to-air missiles do in the 8-10km dogfight[2]. The near space maneuverability of the interceptor is widely believed to be no more than 6-10g[3] or so due to the thin atmosphere. So, three key issues are presented for the interception of the hypersonic weapons: 1) The hypersonic speed of the target, 2) Target active/random maneuverability, 3) Interceptor maneuverability limitation. These factors enables the hypersonic weapon to break through the present defense system more easily than ever. To design a satisfied guidance law for the interceptor of the hypersonic weapon, the above mentioned key factors should be well taken care.

Traditional guidance and control algorithm usually uses the guidance loop as its outer loop and is only responsible for giving overload command. And then the overload command is given to the control loop which is only responsible for tracking this command, eventually achieving the missile's interception toward its target. Although it is always desirable to design the control loop, or the autopilot, to have a better dynamic performance, in actuality, the controller which could be seen as a second order system is inevitable to have phase delay and amplitude attenuation. As a result, the missile always has some unexpected miss distance in the interception. That is one of the most important reasons why an interceptor misses its target.

Recently, some novel guidance and control scheme are proposed[4][5], to deal with the above mentioned issues, and each have their strengths and weaknesses in relation to different application scenarios.

Integrated guidance and control scheme is under heated discussion recent years. Its architecture is shown in Fig. 2. In one way to understand integrated guidance and control (IGC) method is that IGC system combines the guidance and control loops into one loop and gives the rudder deflection command directly to the missile according to the relative motion(Line of Sight rate), which evidently responds more quickly and precisely by avoiding the delay and attenuation caused by the two loops. 


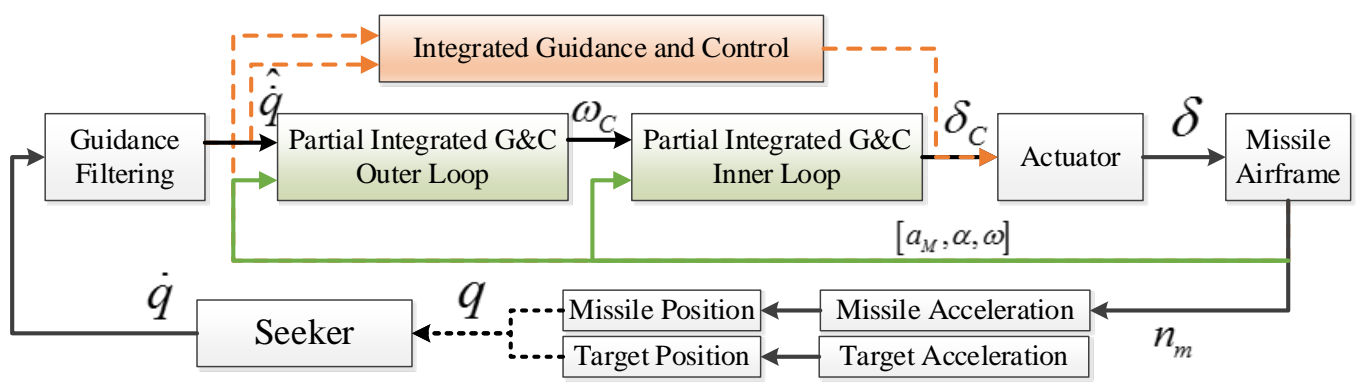

Fig. 1 Integrated/Partial-Integrated guidance and control

\section{Partial Integrated Guidance and Control Scheme}

Partial Integrated Guidance and Control(PIGC) is another new idea of guidance and control design which has been proposed in recent years. Unlike the above mentioned IGC scheme, PIGC is a two loop guidance and control scheme, the outer guidance loop gives pitch rate command to the inner control loop, while the inner loop gives the rudder deflection command to manipulate the missile as shown in the Fig. 1. PIGC is a two loop but not a single loop design is the reason why it is called PARTIAL integrated. Different from separated guidance and control, the outer loop is designed with a full state feedback that is to say the guidance law considers not only the target-missile relative motion, but also the missile inner dynamic, it is an integrated guidance design. This is the reason why it is called partial INTEGRATED. With an inner loop to take care of the inner dynamic of the missile, the stability of the system is more easily to be guaranteed. With a full state feedback, this scheme could be more efficient than the separate guidance and control method.

\section{Model Description}

\section{A. Partial Integrated Guidance and Control Model}

Without loss of generality, we present hereinafter only the subsystems that govern the motion of an interceptor in its longitudinal plane. The planar engagement between the interceptor and its target is shown in Fig. 2:

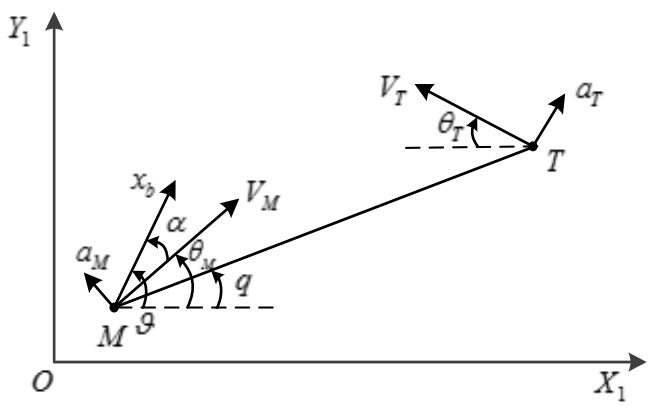

Fig. 2 Engagement geometry

Traditional guidance laws usually regards the missile and its target as mass points, deliberates on the mass point of its rigid motion and ignores the attitude states. By contrast, the partial integrated guidance and control method takes the full dynamic into consideration; therefore, pitch angle $\vartheta$, pitch rate $\omega_{z}$ and angle of attack $\alpha$ of the missile are needed in the design as shown in Fig. 2. The motion equations of the missile in the longitudinal plane are as follows: 


$$
\begin{aligned}
& \ddot{q}=-2 \frac{\dot{r}}{r} \cdot \dot{q}-\frac{C_{Y}^{\alpha} Q S_{r e f}}{m r} \cos \left(\theta_{M}-q\right) \cdot \alpha+\frac{a_{T N}}{r} \cos \left(\theta_{T}+q\right) \\
& r \dot{q}=-V_{M} \sin \left(\theta_{M}-q\right)+V_{T} \sin \left(\theta_{T}+q\right) \\
& \dot{\theta}_{M}=\frac{1}{m V_{M}} C_{Y}^{\alpha} Q S_{r e f} \cdot \alpha \\
& \dot{\alpha}=\omega_{Z}-\frac{1}{m V_{M}} C_{Y}^{\alpha} Q S_{r e f} \cdot \alpha \\
& \dot{\omega}_{Z}=\frac{m_{Z}^{\delta_{Z}} Q S_{r e f} l}{J_{Z}} \cdot \delta_{Z}+\frac{m_{Z}^{\alpha} Q S_{r e f} l}{J_{Z}} \alpha
\end{aligned}
$$

Where the missile's velocity is $V_{M}$ and its flight path angle is $\theta_{M}$; the LOS angle between the missile and its target is $q$; the relative distance between the missile and its target is $r$ the velocity of the target is $V_{T}$ and its flight path angle is $\theta_{T} ; x_{b}$ is the axle of the missile's airframe; the pitch angle is $\vartheta$; the angle of attack is $\alpha$.

Line Of Sight (LOS) $q$, LOS rate $\dot{q}$ and relative range $r$ are basic elements to describe the relative motion of the missile and the target. The kinematic equations are expressed by the following relations:

\section{B. Relative Degree of the Outer Loop}

To obtain the relative degree of the control command $\omega_{z}$ of the partial integrated guidance and control method, we keep on deriving the LOS rate $\dot{q}$ until the explicit formula of its derivative of a certain order contains the control input $\omega_{z}$.

The derivation of $\dot{q}$ produces:

$$
\ddot{q}=\frac{1}{r}\left[-2 \dot{r} \dot{q}-a_{M N} \cos \left(\theta_{M}-q\right)+a_{T N} \cos \left(\theta_{T}+q\right)\right]
$$

The LOS rate is expressed as the derivative of the first order, and does not contain the control input $\omega_{z}$ explicitly. The continuous derivation of the above equation produces:

$$
\begin{aligned}
\dddot{q}= & \frac{1}{r}\left\{-2 \dddot{r} \dot{q}-3 \ddot{r} \ddot{q}-\left[a_{M N}\left(\dot{q}-\dot{\theta}_{M}\right) \sin \left(\theta_{M}-q\right)+\dot{a}_{M N} \cos \left(\theta_{M}-q\right)\right]\right. \\
& \left.-\left[a_{T N}\left(\dot{q}+\dot{\theta}_{T}\right) \sin \left(\theta_{T}+q\right)-\dot{a}_{T N} \cos \left(\theta_{T}+q\right)\right]\right\}
\end{aligned}
$$

Where the LOS rate is expressed as the differentiating of the second order, and the $\dot{a}_{M}$ can be expressed as:

$$
\dot{a}_{M N}=\frac{C_{Y}^{\alpha} Q S_{r e f}}{m} \dot{\alpha}=\frac{C_{Y}^{\alpha} Q S_{r e f}}{m}\left(\dot{\vartheta}-\dot{\theta}_{M}\right)=\frac{C_{Y}^{\alpha} Q S_{r e f}}{m}\left(\omega_{z}-\dot{\theta}_{M}\right)
$$

In the Eq.(4), it can be seen that the control input $\omega_{z}$ appears expressly in the second-order derivative of the control output $\dot{q}$. Therefore, the relative degree of the control input $\omega_{Z}$ is 2 . So we could get the following equation:

$$
\begin{aligned}
& \sigma^{(2)}=q^{(3)}=f_{\omega_{Z}} \cdot \omega_{Z}+\frac{1}{r}\left(f_{1}+f_{2}+f_{3}\right) \\
& f_{\omega_{Z}}=\frac{1}{r} \frac{C_{Y}^{\alpha} Q S_{r e f} \cos \eta_{M}}{m} \\
& f_{1}=-a_{M N}\left(\omega_{Z}-\dot{\theta}_{M}\right) \sin \eta_{M} \\
& f_{2}=\dot{a}_{T N} \cos \left(\theta_{T}+q\right)-a_{T N}\left(\dot{q}+\dot{\theta}_{T}\right) \sin \left(\theta_{T}+q\right) \\
& f_{3}=2 \ddot{r} \dot{q}+3 \dot{r} \ddot{q}
\end{aligned}
$$




\section{Relative Degree of the Inner Loop}

In order to design the inner loop controller, the relative degree of the inner loop must be derived as well. Same as the outer loop, we keep on deriving the pitch rate $\omega_{z}$ until the explicit formula of its derivative of a certain order contains the control input $\delta_{z}$.

In Eq.(20), it is easy to find that $\dot{\omega}_{Z}$ contains $\delta_{z}$. So the relative degree is 1 .

$$
\dot{\omega}_{Z}=\frac{m_{Z}^{\delta_{Z}} Q S_{r e f} l}{J_{Z}} \cdot \delta_{Z}+\frac{m_{Z}^{\alpha} Q S_{r e f} l}{J_{Z}} \alpha
$$

\section{The Outer Loop Controller Design}

Quasi-Continuous High Order Sliding Mode(QCHOSM) control approach is a recent proposed method to deal with high order sliding mode control problems due to its convenient of application.

\section{A. Sliding Mode Manifold Design}

To design the HOSM controller, a sliding manifold must be chosen first. In this design, we try to make the LOS rate converge to a desire LOS rate or a small neighbor domain near the desirer LOS rate, thus ensuring that the missile approaches its target in a proper way. Here we choose the desire LOS rate as zero, so the missile would attack the target in a quasi-parallel way which will lead to a minimal overload requirement. So the sliding manifold is chosen as follows:

$$
\sigma_{1}=\dot{q}_{d}-\dot{q}=0-\dot{q}=-\dot{q}
$$

From the above discussion in Sec. II, we know that the control input in relation to control output $\dot{q}$, namely the relative degree of sliding mode manifold $\sigma_{1}$ is 2 . So the following design will be about a 2nd-order sliding mode controller.

\section{B. Design of the Quasi-Continuous HOSM Controller}

First, Eq. (5)can be expressed as follows:

$$
\ddot{\sigma}_{1}=h_{1}(t, x)+g_{1}(t, x) u_{1}
$$

Where $h_{1}(t, x), g_{1}(t, x), u_{1}$ is expressed as follows:

$$
\begin{aligned}
& h_{1}(t, x)=\frac{1}{r}\left(f_{1}+f_{2}+f_{3}\right) \\
& g_{1}(t, x)=f_{\omega_{Z}} \\
& u_{1}=\omega_{Z}
\end{aligned}
$$

According to the quasi-continuous high order sliding mode control method proposed by Arie Levant in [6], the controller with a sliding mode manifold whose relative degree is 2 should be designed in the following form, where $\beta$ is a control gain term:

$$
u_{1}=-\beta_{1}\left(\left|\dot{\sigma}_{1}\right|+\left|\sigma_{1}\right|^{1 / 2}\right)^{-1}\left(\dot{\sigma}_{1}+\left|\sigma_{1}\right|^{1 / 2} \operatorname{sign} \sigma_{1}\right)
$$

\section{Stability Conditions}

The conditions under which the LOS angular velocity may converge are as follows:

$$
0<K_{m 1} \leq g_{1}(t, x) \leq K_{M 1} \text {, and }\left|h_{1}(t, x)\right| \leq C_{1}
$$

Where $K_{m}, K_{M}$ and $C$ are all larger than zero. This is a proven theorem by Arie Levant in [6]. The system we discussed meets the above requirements and the proof is as follows:

$$
g_{1}(t, x)=f_{\omega_{Z}}=\frac{1}{r} \frac{C_{Y}^{\alpha} Q S_{r e f} \cos \eta_{M}}{m}
$$

The dynamic pressure $Q$ is $Q=\rho V_{M}^{2} / 2$ Where $\rho=0.08803 \mathrm{Kg} / \mathrm{m}^{3}$ (altitude $=20 \mathrm{Km}$ ) is the air density, $V_{M}=2000 \mathrm{~m} / \mathrm{s}$ is the speed of the missile, so $Q$ is always positive. $S_{r e f}=0.26 \mathrm{~m}^{2}$ and 
$l_{\text {ref }}=3.65 \mathrm{~m}$, denotes the reference area and the reference length of the missile, they are both positive constant. $m=100 \mathrm{Kg}$ denotes the missile mass. $r$ is the relative distance, it is always a positive number. $C_{Y}^{\alpha}$ is the lift coefficient caused by the angle of attack, it varies from 0.18 to 0.37 , it is always a positive number.

Meanwhile, considering that the missile under guidance and control is unlikely to fly away from its target, namely the angle between the missile's velocity and its LOS direction cannot be larger than $90^{\circ}$, then:

$$
\left|\theta_{M}-q\right|<\pi / 2 \Rightarrow \cos \left(\theta_{M}-q\right)>0
$$

Summing up the above conditions, then we can get:

$$
g_{1}(t, x)>0
$$

In other words, there is a positive real number $K_{m}$ exists could satisfy the following condition:

$$
0<K_{m 1}<g_{1}(t, x)
$$

Before the missile hits on the target, the term will be positive and limited, then, we can get:

$$
0<K_{m 1}<g_{1}(t, x)<K_{M 1}
$$

With Eq.(13), then:

$$
h_{1}(t, x)=\frac{1}{r}\left(f_{1}+f_{2}+f_{3}\right)
$$

In the practice sense, the changes in both the LOS rate and the acceleration of the missile, the acceleration of the target are limited and continuous. So the following variables $\dot{q}, \ddot{q}, \dddot{q}, \dot{a}_{M}, \dot{a}_{T}$ and $\ddot{a}_{T}$ are all bounded. However, because $h_{1}(t, x)$ contains the item $1 / r$, when the relative distance between the missile and its target is zero, the boundary of $h_{1}(t, x)$ is not guaranteed. In [6], Arie Levant only requires that condition (15) should be locally valid, not requiring that it should be globally valid. Therefore, the integrated guidance and control method is applicable here. So, the above mentioned condition is satisfied with a positive number $C_{1}$ :

$$
\left|h_{1}(t, x)\right| \leq C_{1}
$$

\section{The Arbitrary-Order Robust Exact Differentiator}

The quasi-continuous HOSM control method needs to use the third derivative of the sliding manifold, namely $\ddot{q}$. How to calculate or accurately estimate $\ddot{q}$ is one of the key problems to be solved. We use the Arbitrary-Order Robust Exact Differentiator designed by Arie Levant to differentiate the LOS rate $\dot{q}$, thus obtaining $\ddot{q}$.

The Arbitrary-Order Robust Exact Differentiator can be constructed in accordance with High Order Sliding Modes, Differentiation and Output Feedback Control in Ref. [7].

To obtain the first-order derivative of $\dot{q}$, we construct a second-order sliding mode differentiator and estimate the derivative $\dot{q}$ of each order. In view of differential precision, we configure the following third-order differentiator.

$$
\left\{\begin{array}{l}
\dot{z}_{0}=v_{0}, v_{0}=-\lambda_{0} L^{1 / 3}\left|z_{0}-\dot{q}\right|^{2 / 3} \operatorname{sign}\left(z_{0}-\dot{q}\right)+z_{1}, \\
\dot{z}_{1}=v_{1}, v_{1}=-\lambda_{1} L^{1 / 2}\left|z_{1}-v_{0}\right|^{1 / 2} \operatorname{sign}\left(z_{1}-v_{0}\right)+z_{2}, \\
\dot{z}_{2}=v_{2}, v_{2}=-\lambda_{2} L \operatorname{sign}\left(z_{2}-v_{1}\right),
\end{array}\right.
$$

Where $z_{2}, z_{1}, z_{0}$ are the estimations of $\dddot{q}, \ddot{q}, \dot{q}$. 


\section{The Inner Loop Controller Design}

To design a proper inner loop controller, as we have done in the Section III, we should first choose a sliding manifold. Although the relative degree of the inner loop is one, high order sliding mode controller is still needed to implement a virtual control design to alleviate the chattering.

\section{A. Sliding Mode Manifold Design}

The main purpose of the inner loop is to track the outer loop's pitch rate command, so we design the following sliding manifold:

$$
\sigma_{2}=\omega_{d}-\omega_{z}
$$

$\omega_{d}$ is the desired pitch rate command given by outer loop, $\omega_{Z}$ is the real pitch rate feedback from the sensor. By maintaining the sliding manifold moving around the zero point, the pitch rate of the missile could track the pitch rate command.

\section{B. Design of the Quasi-Continuous HOSM Controller}

First, Eq. (10)can be expressed as follows:

$$
\dot{\sigma}_{2}=h_{2}^{\prime}(t, x)+g_{2}^{\prime}(t, x) u_{2}
$$

Where $h_{2}(t, x), g_{2}(t, x), u_{2}$ is expressed as follows:

$$
\begin{aligned}
& h_{2}^{\prime}(t, x)=\frac{m_{Z}^{\alpha} Q S_{r e f} l}{J_{Z}} \alpha \\
& g_{2}^{\prime}(t, x)=\frac{m_{Z}^{\delta_{Z}} Q S_{r e f} l}{J_{Z}} \\
& u_{2}^{\prime}=\delta_{Z}
\end{aligned}
$$

In this situation, we could implement a first order Quasi-Continuous HOSM controller as the following:

$$
u_{2}^{\prime}=-\alpha_{2}^{\prime} \operatorname{sign}(\sigma)
$$

But as for the practical sense, a first order sliding mode controller in this form would result in a serious chattering phenomenon, which would surely be harmful to the system and sometimes even be taken as unacceptable. There are many researchers who have done lots of work on the avoidance of the chattering phenomenon. Researchers ${ }^{[7][8]}$ has proposed several saturation functions to replace the signal functions to build a boundary layer to alleviate the chattering, some researchers ${ }^{[9]}$ turn to use the fuzzy control scheme to alleviate with the chattering. But to the authors' knowledge, none of the proposed method have theoretically proved the robustness of the revised controller is maintained. In this work, we will introduce the idea of virtual control to deal with this problem.

\section{Virtual Control Design}

In this paper, the idea of virtual control is introduced to deal with the chattering phenomenon in the controller design of the inner loop.

The idea of virtual control is to artificially increase the relative degree of the system once and to implement a higher order controller $\dot{\delta}_{C}$ on the extended system. And then, the real control $\delta_{C}$ acts on the missile is obtained by integrating the virtual control $\dot{\delta}_{C}$. The integral element will move the relative degree of the control signal to a proper level to act as the rudder deflection command and meantime the rudder deflection command would be smooth enough to act on the missile. In one word, an integral element is introduced by the by introducing a higher order Quasi-Continuous sliding mode controller. 


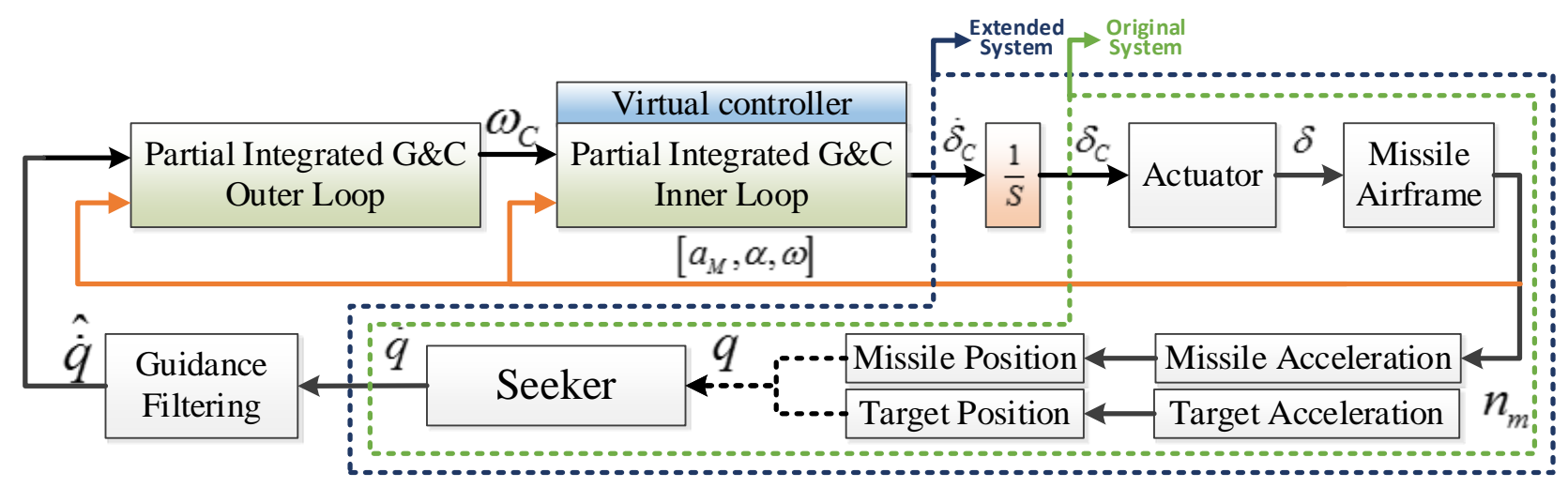

Fig. 3 Partial integrated guidance and control scheme with virtual control

To increase the relative degree of the system, derivation of the Eq.(10) provides the following equation:

$$
\begin{aligned}
& \ddot{\omega}_{Z}=\frac{m_{Z}^{\delta_{Z}} Q S_{r e f} l}{J_{Z}} \cdot \dot{\delta}_{Z}+\frac{m_{Z}^{\alpha} Q S_{r e f} l}{J_{Z}} \dot{\alpha} \\
& \ddot{\sigma}_{2}=h_{2}(t, x)+g_{2}(t, x) u_{2}
\end{aligned}
$$

It is easy to find that when $\dot{\delta}_{z}$ becomes the new control signal the relative degree raise to 2 . Where $h_{2}(t, x), g_{2}(t, x), u_{2}$ is expressed as follows:

$$
\begin{aligned}
& h_{2}(t, x)=\frac{m_{Z}^{\alpha} Q S_{r e f} l}{J_{Z}} \dot{\alpha}=\frac{m_{Z}^{\alpha} Q S_{r e f} l}{J_{Z}}\left(\omega_{\mathrm{Z}}-\dot{\theta}\right) \\
& g_{2}(t, x)=\frac{m_{Z}^{\delta_{Z}} Q S_{r e f} l}{J_{Z}} \\
& u_{2}=\dot{\delta}_{Z}
\end{aligned}
$$

Here we would like to design a $\dot{\delta}_{C}$ controller for the system which the relative degree is 2 .

$$
u_{2}=-\beta_{2}\left(\left|\dot{\sigma}_{2}\right|+\left|\sigma_{2}\right|^{1 / 2}\right)^{-1}\left(\dot{\sigma}_{2}+\left|\sigma_{2}\right|^{1 / 2} \operatorname{sign} \sigma_{2}\right)
$$

The conditions under which the LOS rate may converge are as follows:

$$
0<K_{m 2} \leq g_{2}(t, x) \leq K_{M 2} \text {, and }\left|h_{2}(t, x)\right| \leq C_{2}
$$

Where $K_{m}, K_{M}$ and $C$ are all larger than zero. The system we discussed meets the above requirements and the proof is same as the outer loop design.

\section{The Arbitrary-Order Robust Exact Differentiator}

To obtain the derivative of the $\sigma_{2}$ signal, AORED designed in Sec. III could be used here again, the form is as following:

$$
\left\{\begin{array}{l}
\dot{y}_{0}=w_{0}, w_{0}=-\mu_{0} L_{2}^{1 / 3}\left|y_{0}-\sigma_{2}\right|^{2 / 3} \operatorname{sign}\left(y_{0}-\sigma_{2}\right)+y_{1}, \\
\dot{y}_{1}=w_{1}, w_{1}=-\mu_{1} L_{2}^{1 / 2}\left|y_{1}-w_{0}\right|^{1 / 2} \operatorname{sign}\left(y_{1}-w_{0}\right)+y_{2}, \\
\dot{y}_{2}=w_{2}, w_{2}=-\mu_{2} L_{2} \operatorname{sign}\left(y_{2}-w_{1}\right),
\end{array}\right.
$$

Where $y_{2}, y_{1}, y_{0}$ are the estimations of $\ddot{\sigma}_{2}, \dot{\sigma}_{2}, \sigma_{2}$ here.

\section{Simulation Results}

To verify the HOSM-PIGC method, we compare it with the baseline separated guidance and control. Numerical simulations are designed in three typical engagement scenarios. 
AORED Parameters: the initial value $\lambda_{0}=\lambda_{1}=\lambda_{2}=\lambda_{3}=50 ; z_{0}=0.1 ; z_{1}=z_{2}=z_{3}=0 ; L=1400$; $\mu_{0}=\mu_{1}=\mu_{2}=\mu_{3}=50 ; y_{0}=0.1 ; y_{1}=y_{2}=y_{3}=0 ; L_{2}=1400$; the simulation step is 0.0001second. In this Scenario, the target maneuvers by $a_{T}=40 \sin (\pi t / 2)$. OSMG with three-loop autopilot is introduced as comparison.

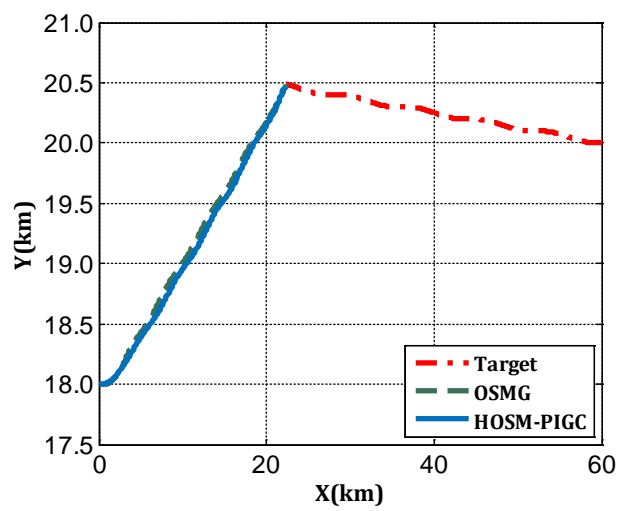

Fig. 4 The trajectories of the missile and its target

To see from Fig. 4 the trajectories of the missile under the two guidance scheme does not show much differences, but the final miss distance differs.

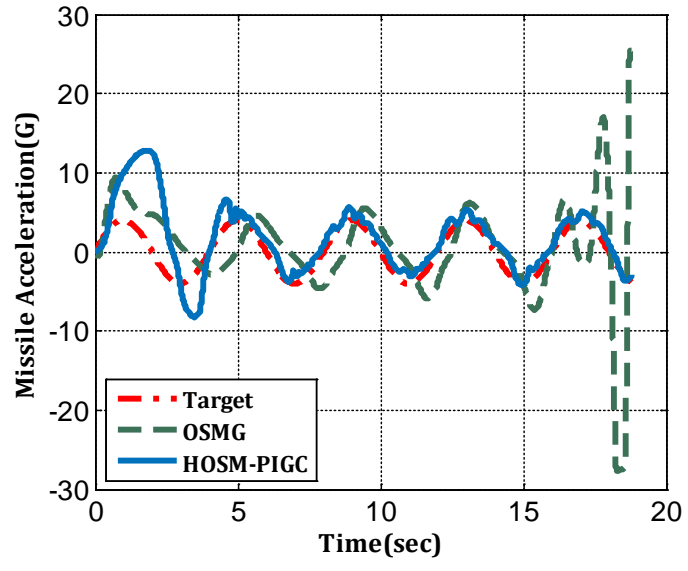

Fig. 5 Missile acceleration profile

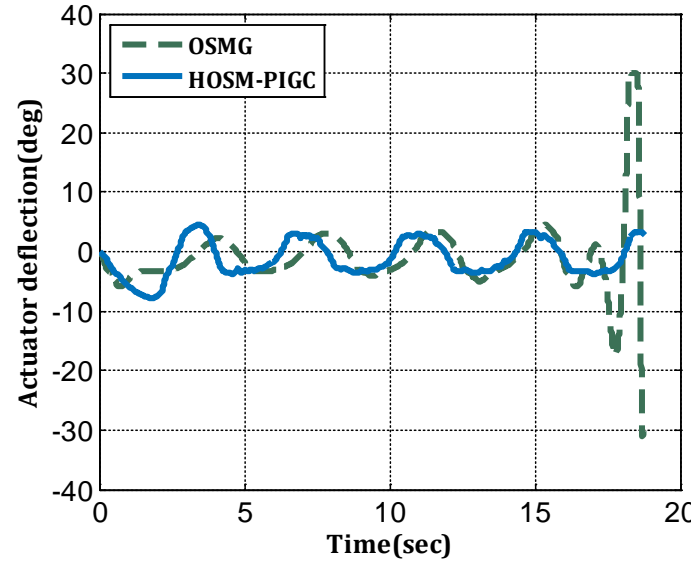

Fig. 6 Actuator deflection

The acceleration profile in Fig. 5 shows the inherit differences between the two scheme. For the first 5 seconds, the two guidance scheme devotes their effort to adjust the initial boresight, and then, the two scheme command the missile to track the target's maneuver acceleration, but the HOSM-PIGC scheme sees a faster convergence speed and a higher precision. On the contrary, the acceleration of the OSMG scheme tracks the target with a continuing phase lag. At the ending phase, the OSMG acceleration fluctuates drastically and diverges at the last few seconds. While the HOSM-PIGC method still maintains the tracking precision which will surly results a smaller miss distance. The actuator deflection curve in Fig.6 also indicates the differences between the two schemes, the HOSM-PIGC keeps a rather stable acceleration command while the OSMG scheme turns to diverge at last.

The final results of the miss distance in this comparison also show great difference of the two scheme. For the OSMG, the 50 simulations average miss distance is $2.35 \mathrm{~m}$. For the HOSM-PIGC, the 50 simulations average miss distance is $0.85 \mathrm{~m}$, which satisfies the interception mission perfectly. 


\section{Conclusions}

To solve a hypersonic target interception problem this paper has proposed a Partial Integrated Guidance and Control method designed by using the Quasi-continuous High Order Sliding Mode approach. The proposed guidance and control method shows some important characters: 1) The command provided by this method is barely affected by the target speed. 2) The proposed method enables the interceptor to achieve a satisfied precision within limited maneuverability. 3) The provided command does not diverge with the LOS rate or the decrease of the relative distance. In addition, the actuator deflection profiles indicates that the proposed virtual control idea works perfectly to alleviate the chattering phenomena without loss of the robustness. In the implementation of the Quasi-continuous HOSM controller, the AORED also provided satisfied precision which not only simplified the calculation but also guarantees the convergence of the HOSM method.

\section{References}

[1] 1. J Xiao,J Yang,J Xue. "Study on the airborne based interception of the hypersonic missiles". Cruise Missile 04. (2015): 13-17.

[2] Sanders, Andrew D., et al. "Multi-Disciplinary Design of a Supersonic, Long-Range, Air-Superiority Missile Through Parametric Design Space Exploration and Physics-Based Modeling." 50th AIAA/ASME/SAE/ASEE Joint Propulsion Conference. 2014.

[3] Lei, Sun, Huang Kexi, Chang Xiaofei, Yan Jie. "Research of Interception of Near-Space Hypersonic Cruise Missile". Joumal of Northwestern Polytechnical University 33.4 (2015): 615-620.

[4] Yan, Han, and Haibo Ji. "Integrated guidance and control for dual-control missiles based on small-gain theorem." Automatica 48.10 (2012): 2686-2692.

[5] Wang, Xianghua, and Jinzhi Wang. "Partial integrated guidance and control for missiles with three-dimensional impact angle constraints." Journal of Guidance, Control, and Dynamics 37.2 (2014): 644-657.

[6] Levant, Arie. "Homogeneity approach to high-order sliding mode design." Automatica 41.5 (2005): 823-830.

[7] Levant, Arie. "Higher-order sliding modes, differentiation and output-feedback control." International journal of Control 76.9-10 (2003): 924-941.

[8] Liang, Yew-Wen, et al. "Robust guidance law via integral-sliding-mode scheme." Journal of Guidance, Control, and Dynamics 37.3 (2014): 1038-1042.

[9] Wang, Liang, Yongzhi Sheng, and Xiangdong Liu. "Continuous time-varying sliding mode based attitude control for reentry vehicle." Proceedings of the Institution of Mechanical Engineers, Part G: Journal of Aerospace Engineering (2014): 0954410014529421.

[10]Roopaei, M., and M. Zolghadri Jahromi. "Chattering-free fuzzy sliding mode control in MIMO uncertain systems." Nonlinear Analysis: Theory, Methods \& Applications 71.10 (2009): 4430-4437. 